Supporting Information

\title{
Design rules for one-step seeded growth of nanocrystals: Threading the needle between secondary nucleation and ripening
}

\author{
Haoran Yang ${ }^{\dagger}$, Leslie S. Hamachi ${ }^{\ddagger}$, Iva Rreza ${ }^{\ddagger}$, Wesley Wang ${ }^{\S}$, Emory M. Chan ${ }^{*}{ }^{\dagger}$ \\ † The Molecular Foundry, Lawrence Berkeley National Laboratory, Berkeley, California \\ 94720, United States \\ ${ }^{\star}$ Department of Chemistry, Columbia University, New York, New York 10027, United States \\ $\S$ Department of Chemistry, University of California at Berkeley, Berkeley, California 94720, \\ United States
}

Table of contents

I. Experimental reaction conditions 1

II. Supplementary figures 2

III. Error analysis of the estimation of size and particle number density 13

$\begin{array}{ll}\text { IV. Reaction simulations } & 14\end{array}$ 


\section{Experimental reaction conditions}

Table S1. A summary of reaction conditions investigated in this study and the corresponding reaction rate constants measured

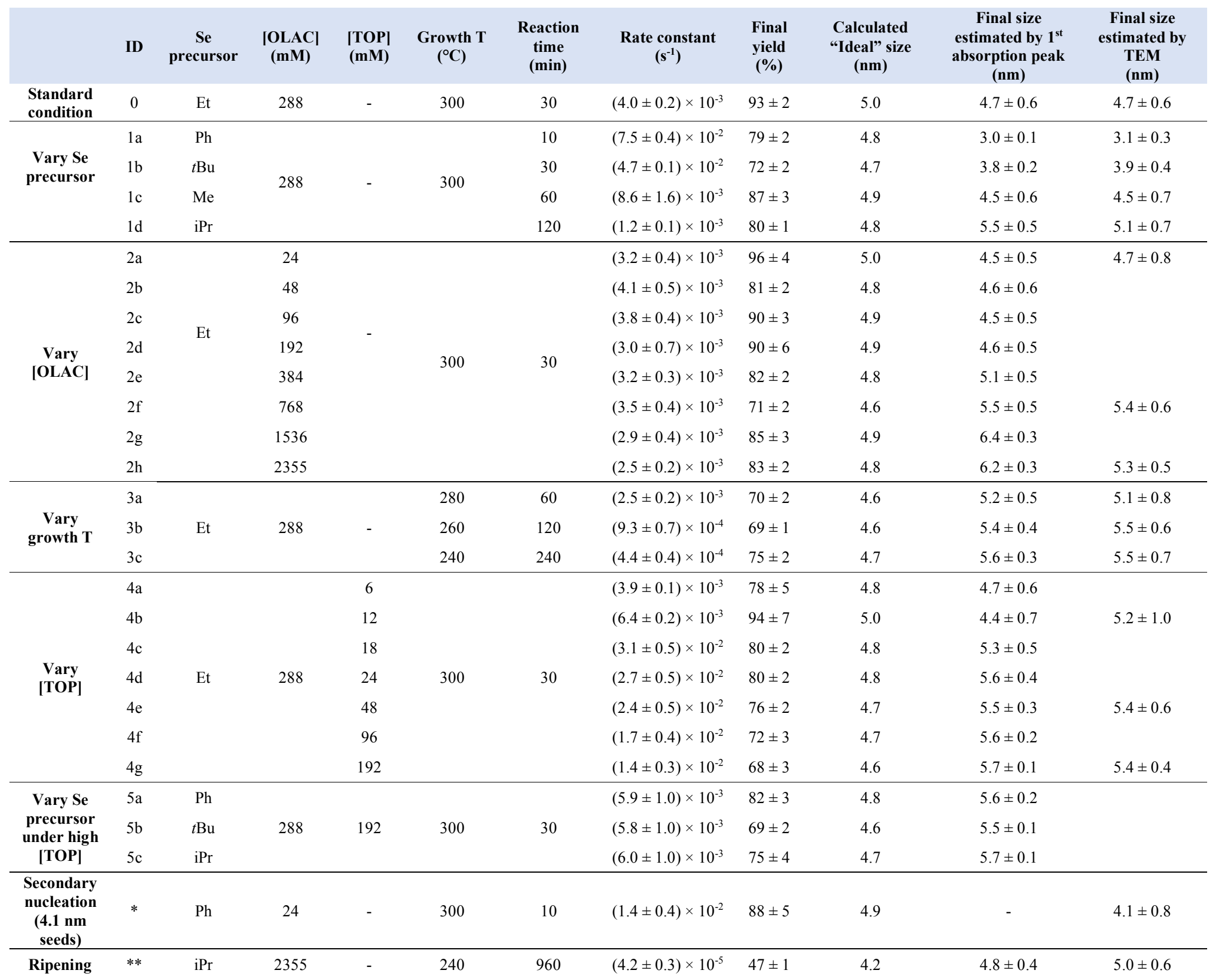




\section{Supplementary figures}

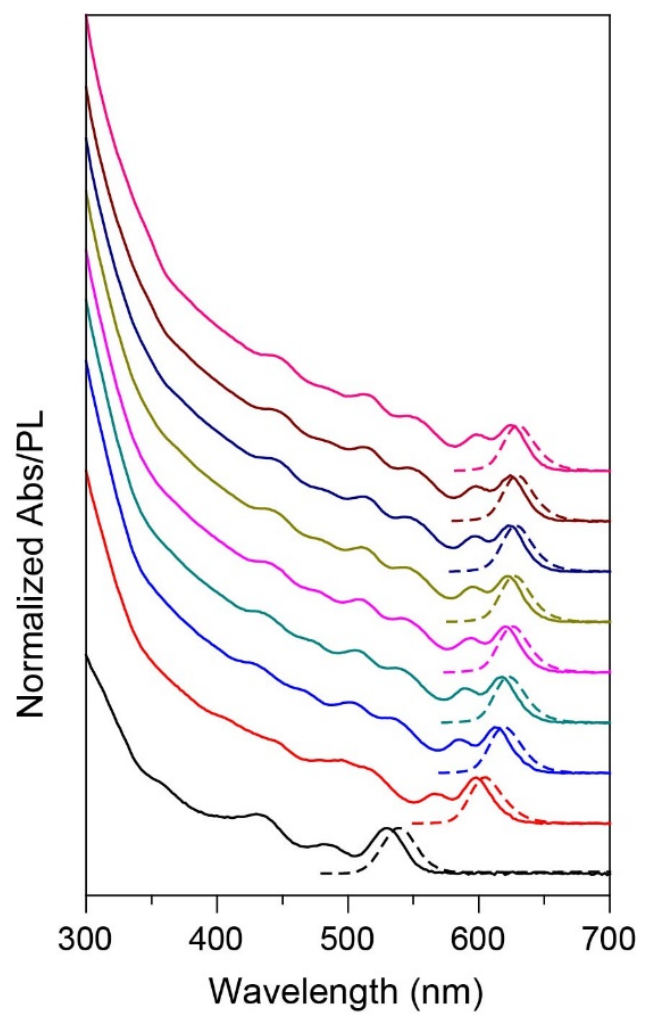

Figure S1. The typical spectral evolution of the CdSe QDs in one seeded growth reaction. Solid line represents absorbance, while dashed line represents photoluminescence intensity. 
(a)
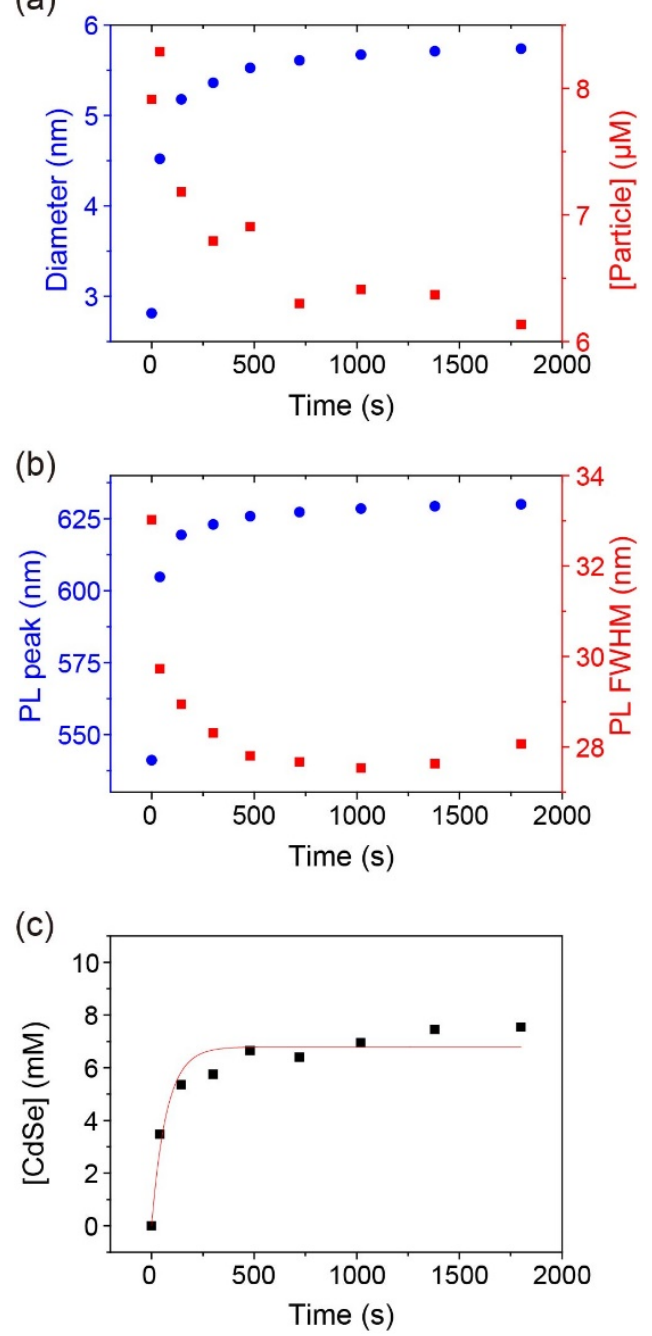

Figure S2. The kinetic information extracted from the UV-Vis and PL spectra of one representative reaction. (a) The temporal evolution of the diameter and particle number density; (b) The temporal evolution of the emission peak wavelength and the corresponding FWHM; (c) The temporal evolution of the molar concentration of CdSe generated by reaction, which is fitted to a ${ }^{\text {st }}$ order rate equation. 

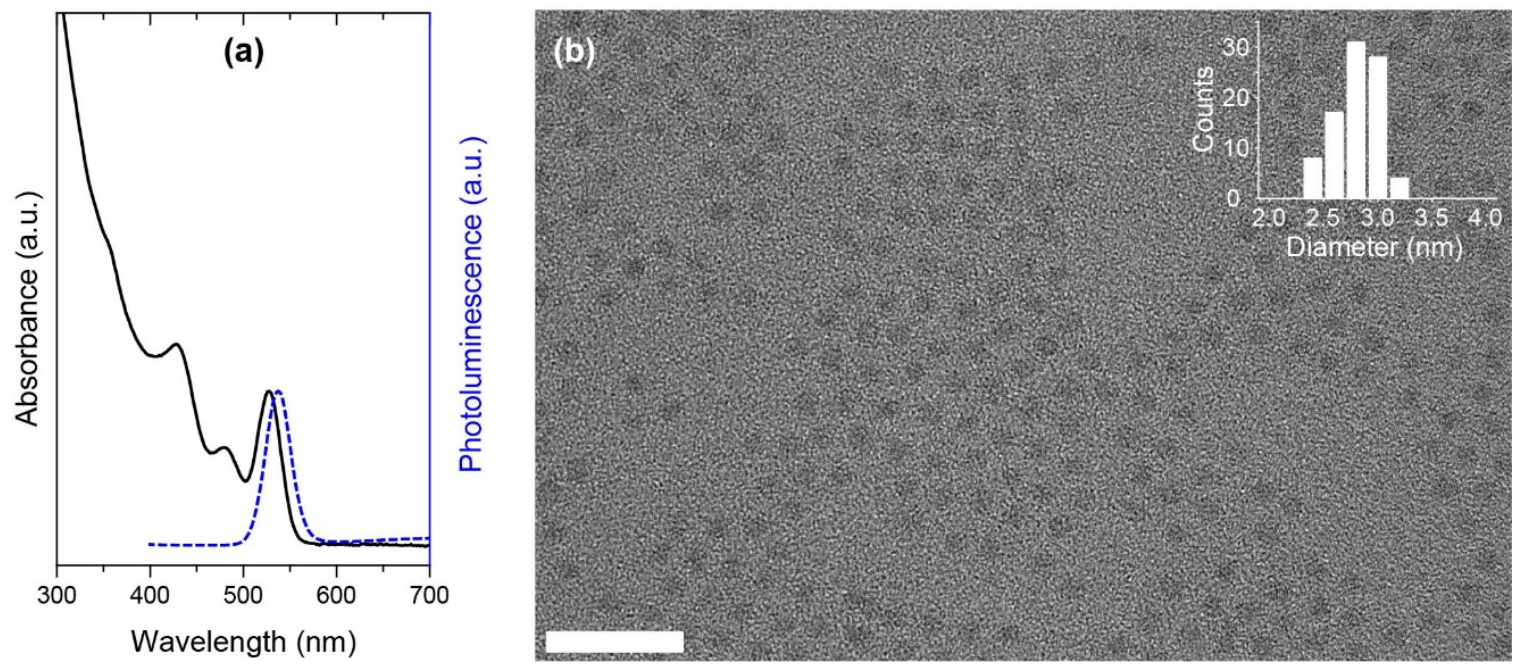

Figure S3. Characterization of the seed CdSe QDs. (a) Absorption and photoluminescence spectrum, (b) TEM image and size histogram. Scale bar represents $20 \mathrm{~nm}$. 

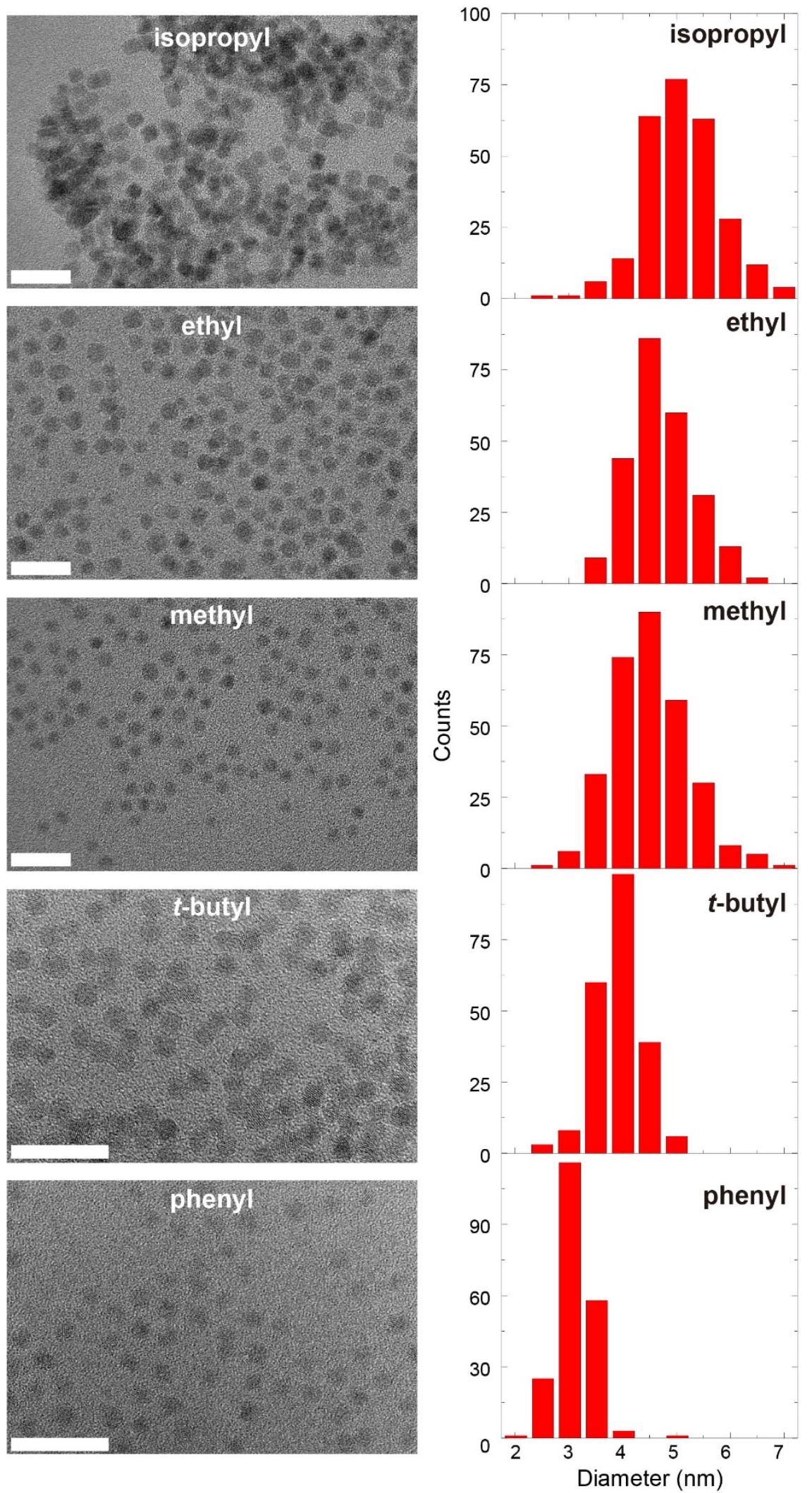

Figure S4. A comparison of the TEM images of the CdSe quantum dots obtained when the selenium precursor is changed, and the corresponding size histograms. Scale bars represent $20 \mathrm{~nm}$. 

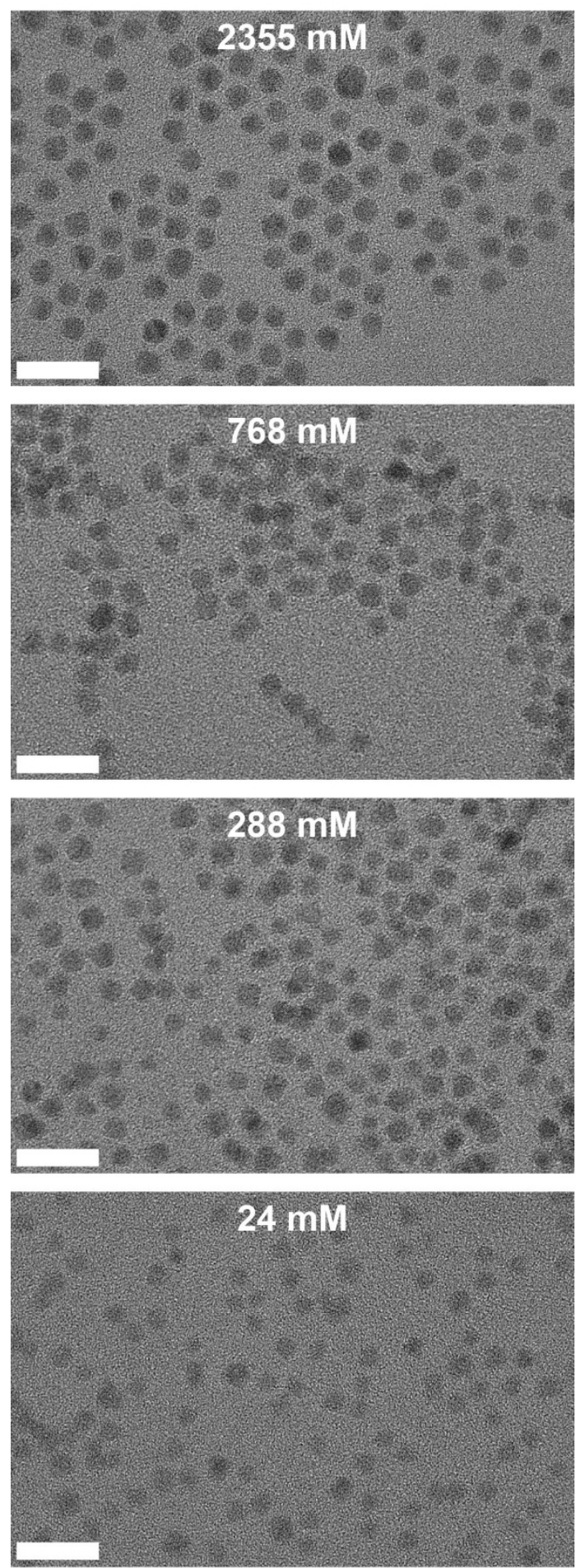

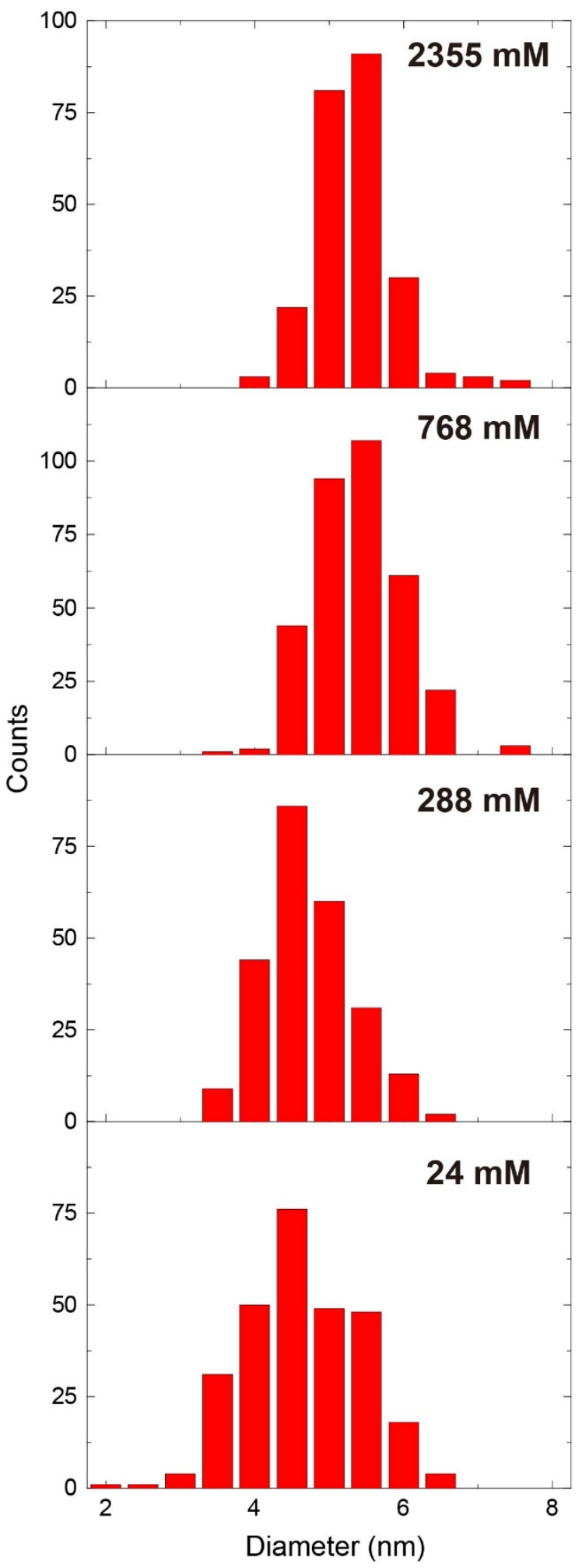

Figure S5. A comparison of the TEM images of the CdSe quantum dots obtained when the concentration of oleic acid is changed, and the corresponding size histograms. Scale bars represent $20 \mathrm{~nm}$. 

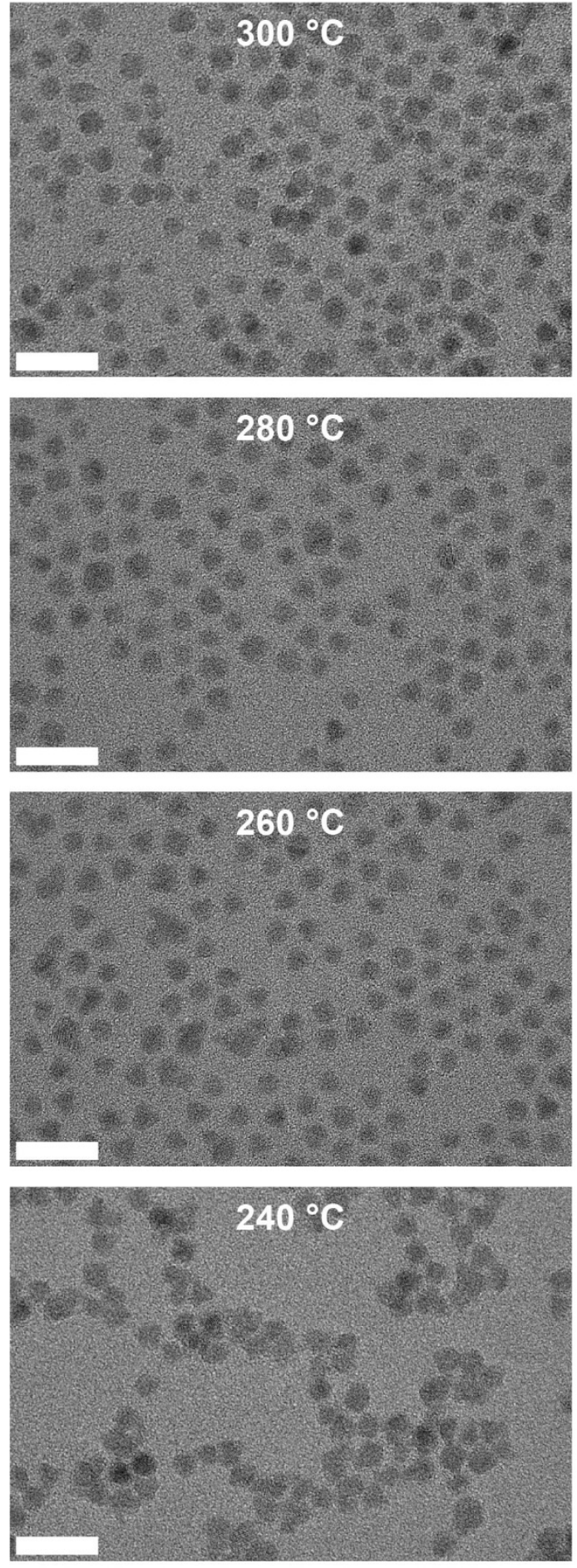

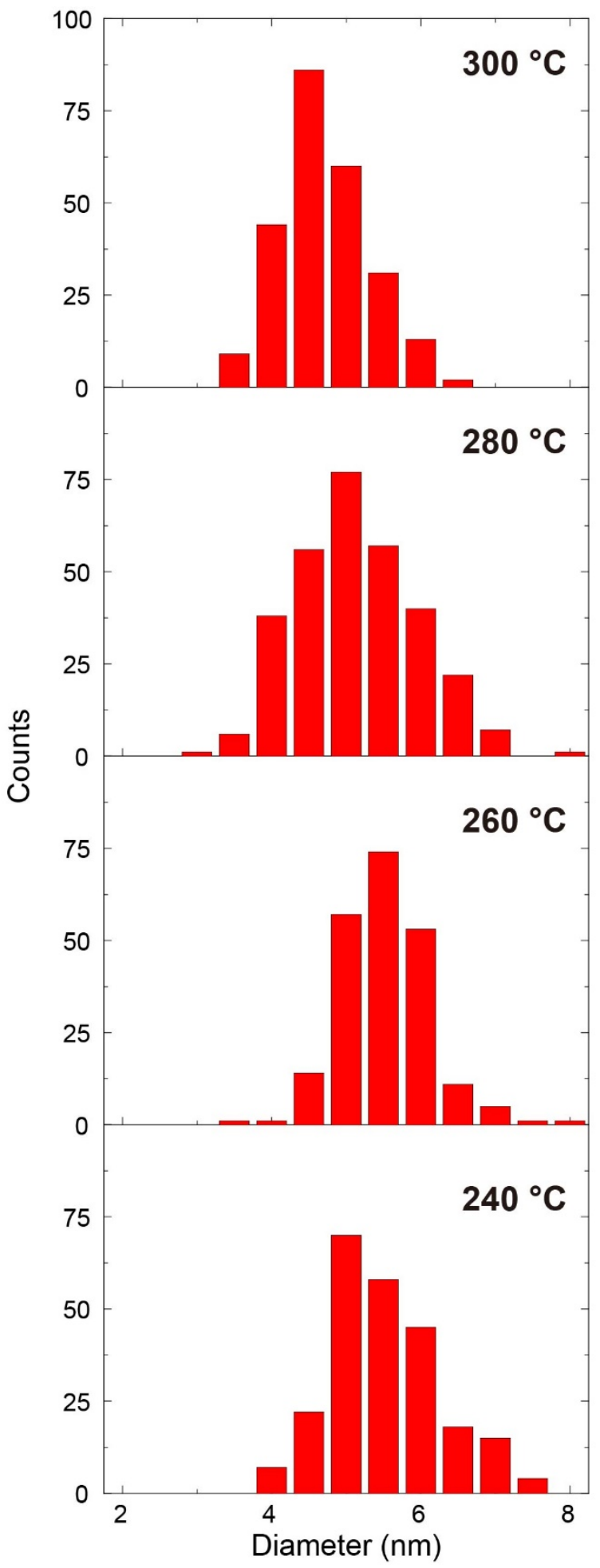

Figure S6. A comparison of the TEM images of the CdSe quantum dots obtained when the growth temperature is changed, and the corresponding size histograms. Scale bars represent $20 \mathrm{~nm}$. 

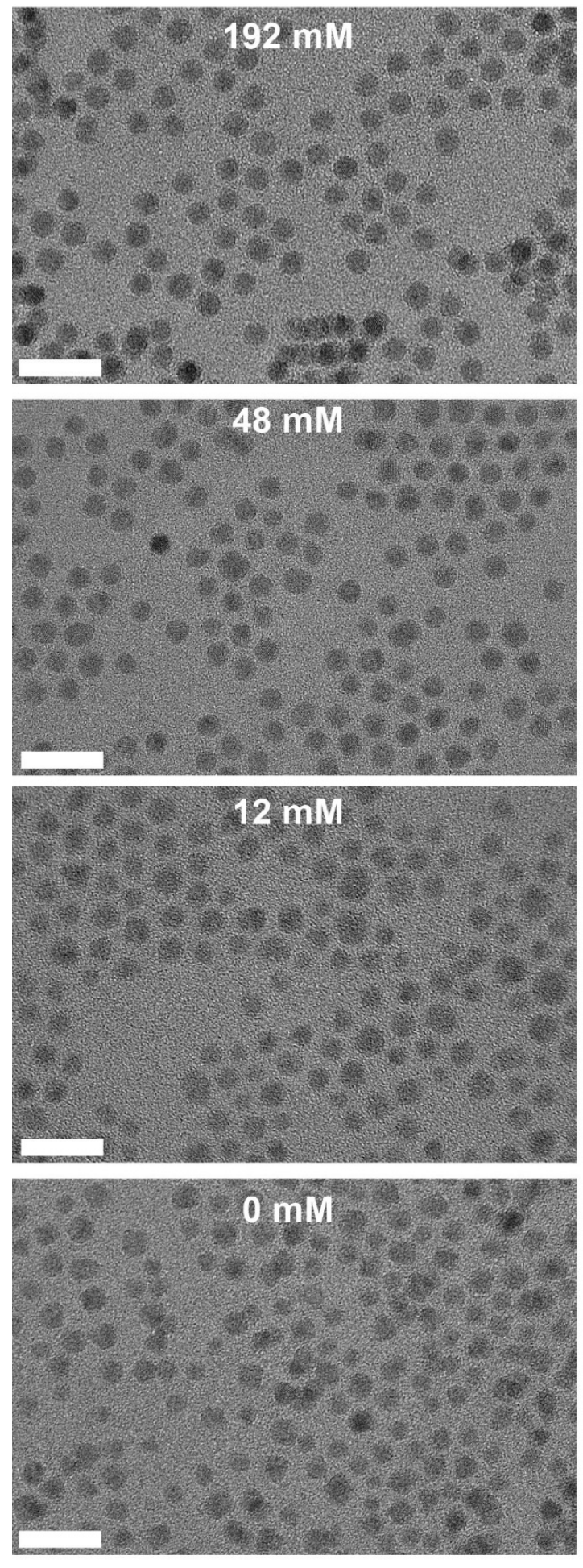

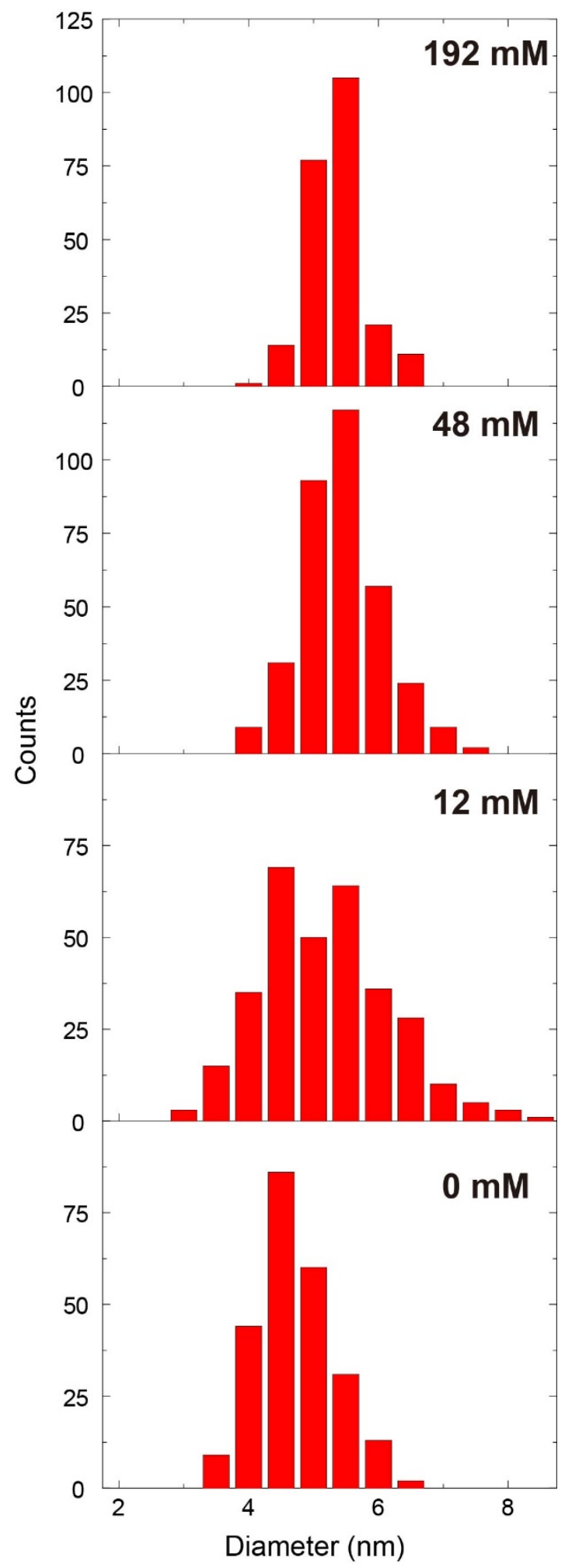

Figure S7. A comparison of the TEM images of the CdSe quantum dots obtained when the concentration of tri- $n$-octylphosphine is changed, and the corresponding size histograms. Scale bars represent $20 \mathrm{~nm}$. 

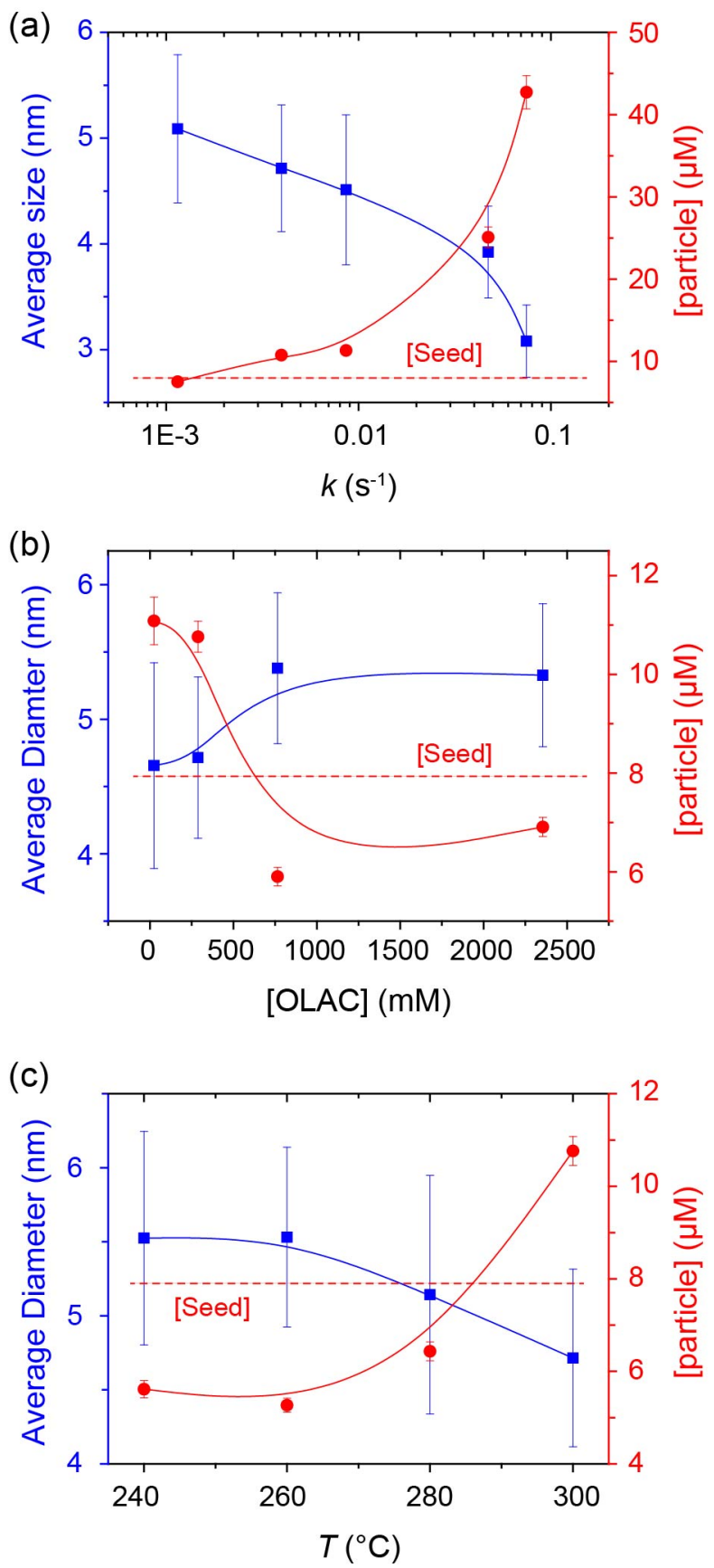

Figure S8. The experimental final diameter and particle number density estimated based on TEM imaging when precursor reaction rate constant $k(a)$, concentration of oleic acid [OLAC] (b), and growth temperature $T$ (c) are varied. The data reported here agree well with the data obtained through the optical method shown in Figure 2. 

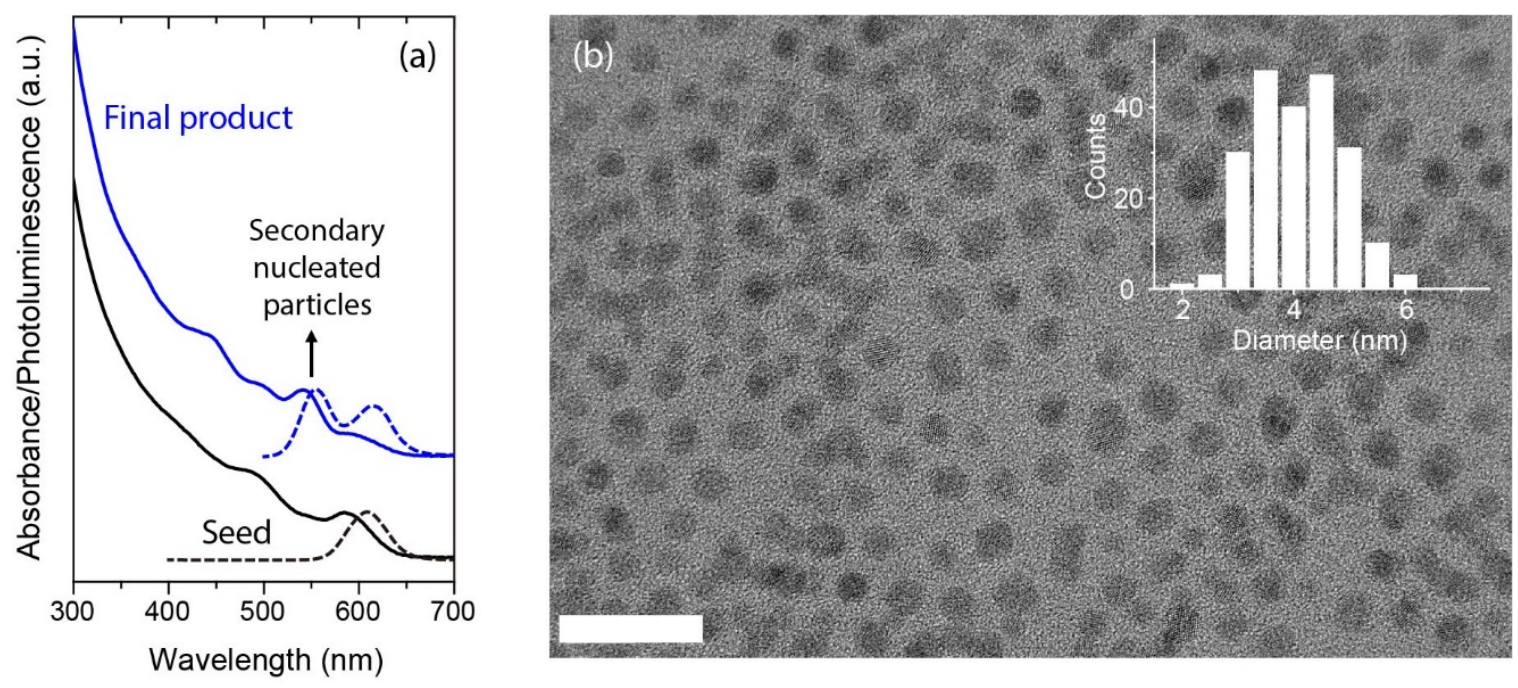

Figure S9. Secondary nucleation revealed in a control experiment (Reaction * in Table S1) in which $4.1 \mathrm{~nm}$ CdSe QDs were used as seed. (a) Absorption and photoluminescence spectrum of the seed and the final product, (b) TEM image and size histogram of the final product. Scale bar represents $20 \mathrm{~nm}$.
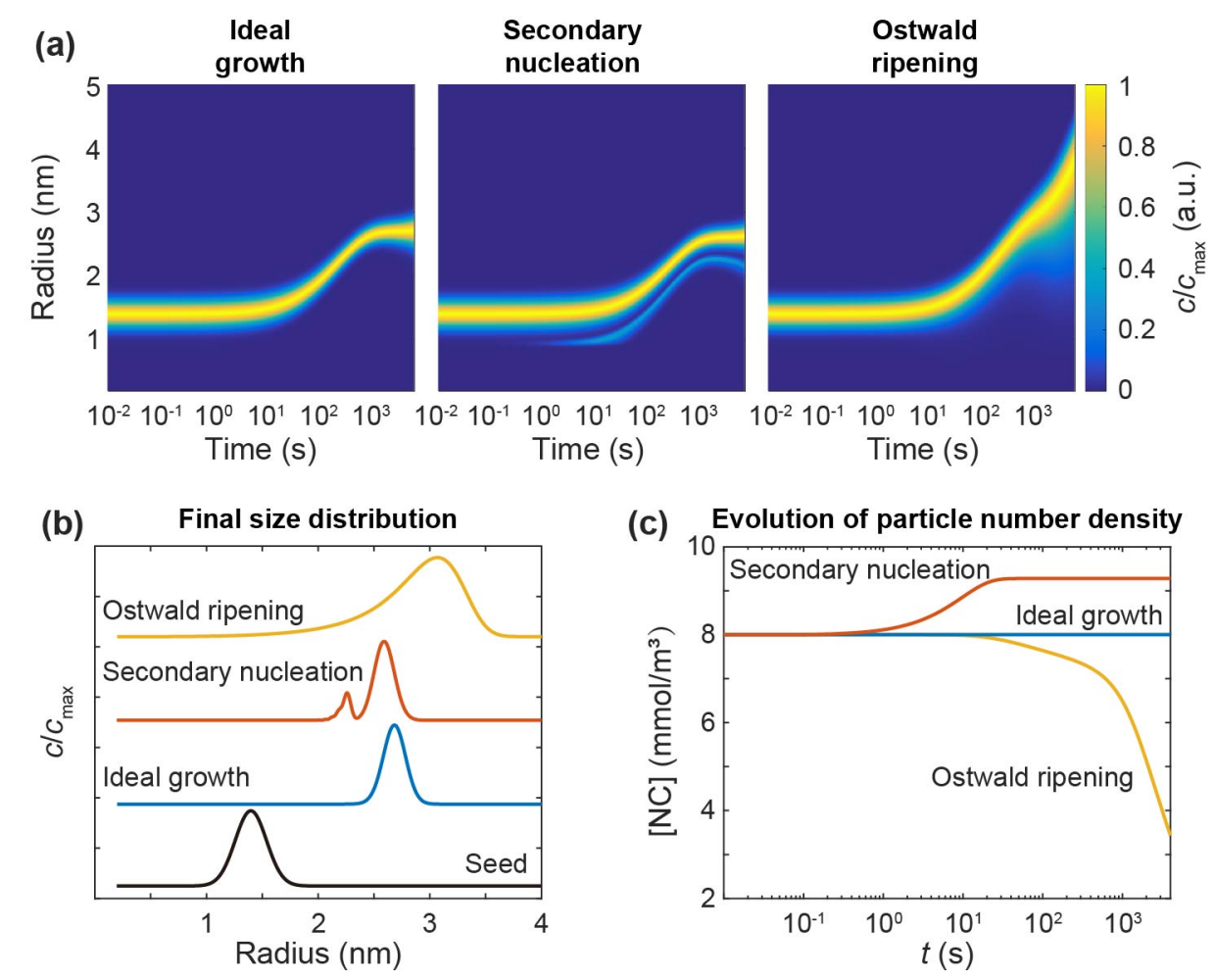

Figure S10. Simulation results. (a) The typical temporal evolution of the size distribution function under three scenarios; (b) a comparison of the final size distribution function (yield $=99 \%$ ); (c) a comparison of the temporal evolution of the particle number density. 


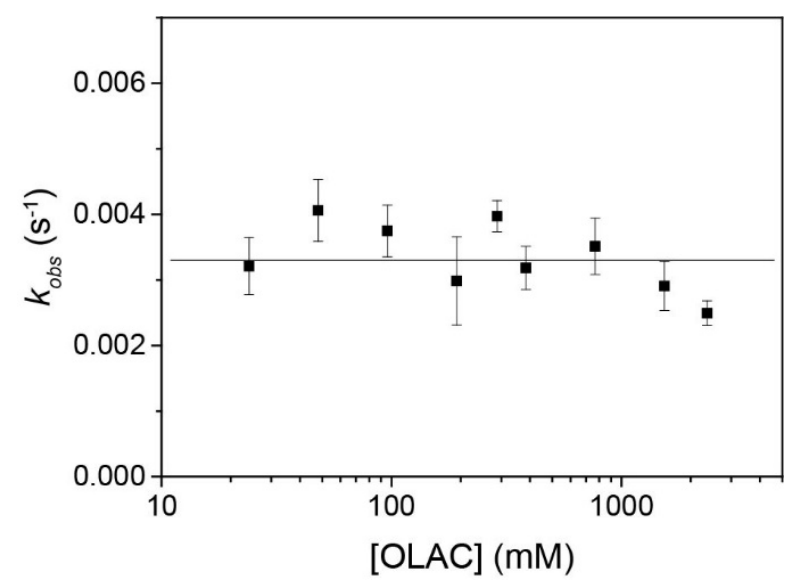

Figure S11. The measured rate constant as a function of oleic acid concentration using 1,3diethylimidazolidine-2-selone at $300{ }^{\circ} \mathrm{C}$.

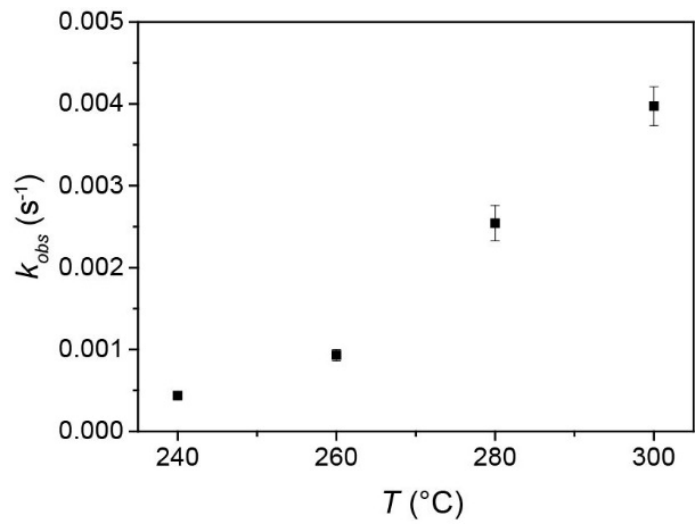

Figure S12. The measured rate constant as a function of temperature using 1,3diethylimidazolidine-2-selone at $[\mathrm{OLAC}]=288 \mathrm{mM}$.
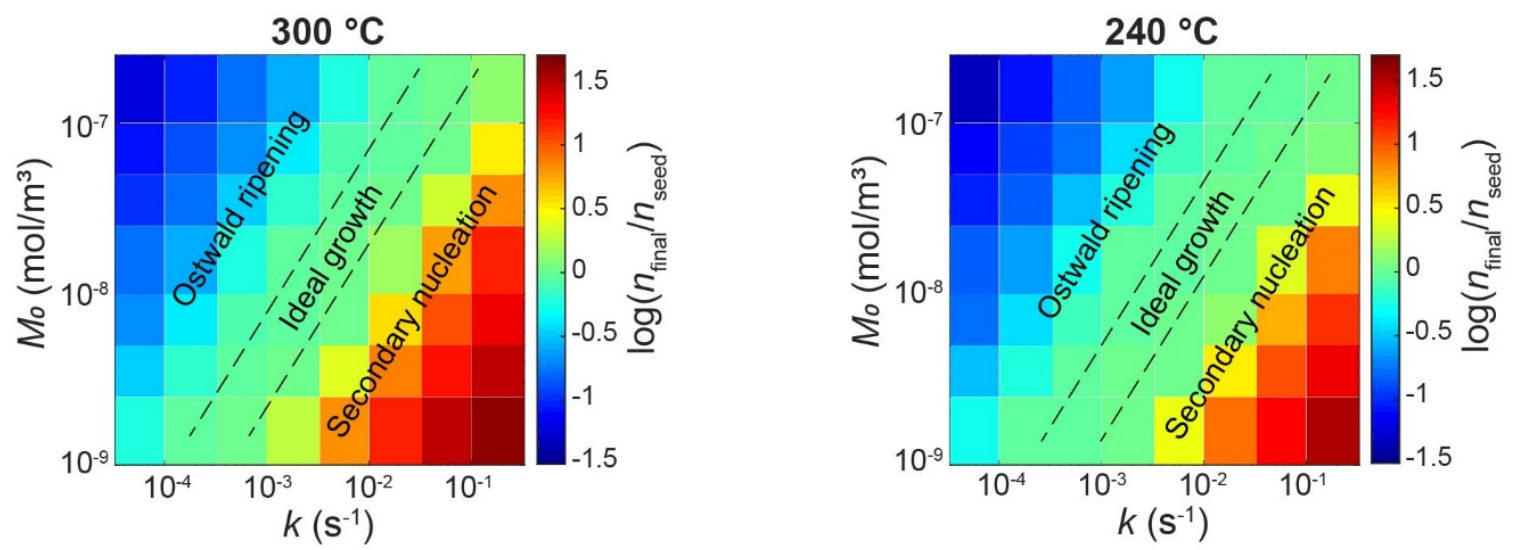

Figure S13. Simulated final particle number $n$ (represented by color) as a function of precursor reaction rate constant and bulk monomer solubility $M_{0}$ 


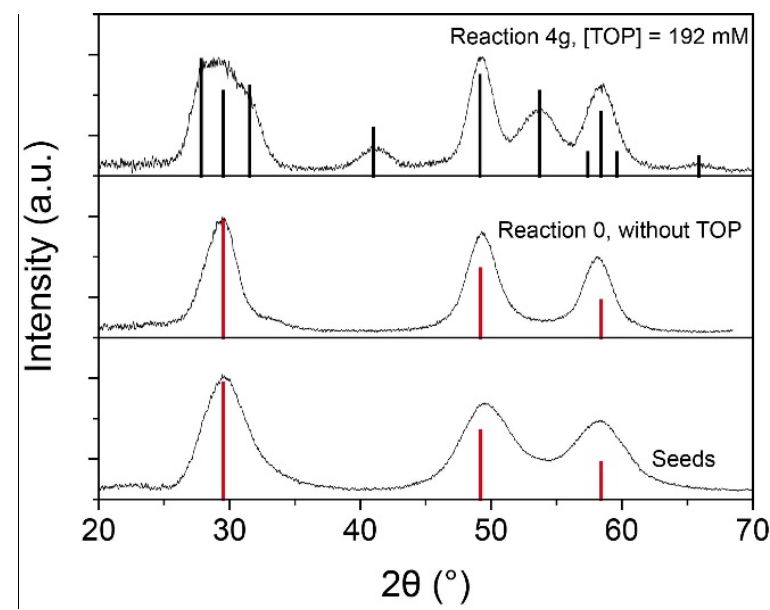

Figure S14. XRD pattern of the representative samples. The vertical lines represent the standard for zincblende (PDF\#19-0191, red lines) and wurtzite (PDF\#08-0459, black lines) CdSe.

(a)

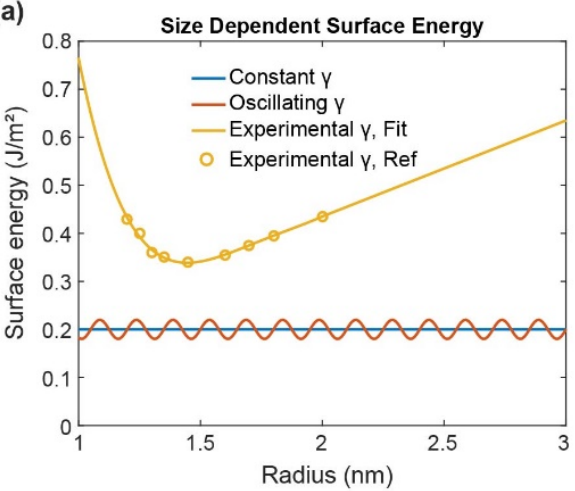

(c)

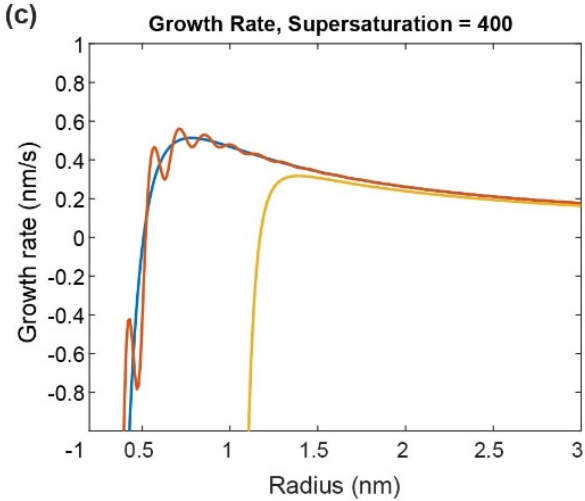

(b)

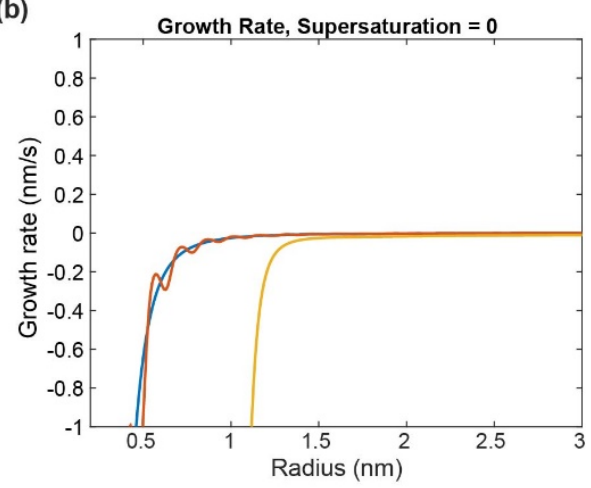

(d)

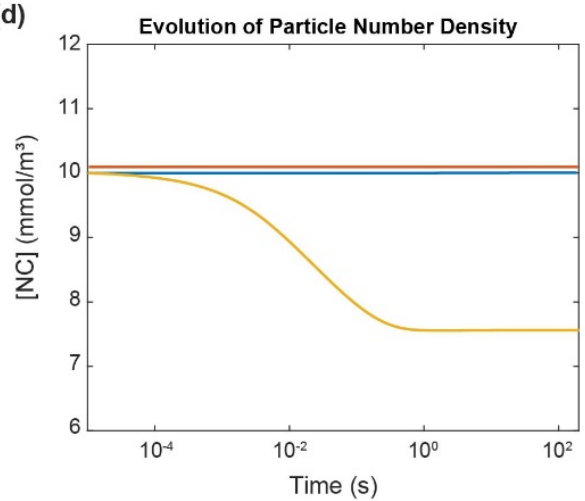

Figure S15. Simulation under three different surface energy profiles. (a) The size dependence of the surface energy per unit area; (b-c) The particle growth rate as a function of particle radius for supersaturation $=0(\mathrm{~b})$ and supersaturation $=400$; (d) The temporal evolution of particle number density, note that the red curve and blue curve are overlapping with each other, and the red curve is intentionally shifted up by $0.1 \mathrm{~mol} / \mathrm{m}^{3}$ so that it is visible. 


\section{Error analysis of the estimation of size and particle number density}

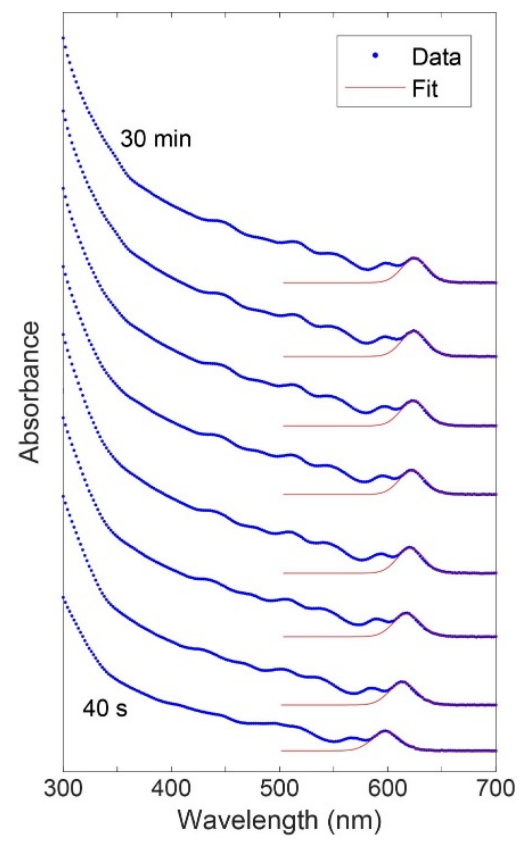

Figure S16. An example of fitting of the first excitonic peak of the absorption spectra to determine the peak position and absorbance maxima.

To estimate the size and particle number density of the CdSe nanoparticles, we first find out the peak positions and the maxima of the first excitonic absorption peak of the absorption data by fitting the longer wavelength half of the peak. An example of the fitting was shown in Figure 16. The average particle size was then estimated by the following empirical formula ${ }^{22}$ for $\mathrm{CdSe}$ nanoparticles:

$$
\mathrm{D}(\mathrm{nm})=59.60816-0.54736 \lambda+1.8873 \times 10^{-3} \lambda^{2}-2.85743 \times 10^{-6} \lambda^{3}+1.62974 \times 10^{-9} \lambda^{4}
$$

where $\lambda$ is the wavelength $(\mathrm{nm})$ of the first absorption maximum. The particle number density was estimated according to the Beer-Lambert Law using the molar extinction coefficient $\left(\varepsilon_{1 S}\right)$ calculated from the following empirical formula ${ }^{22}$ :

$$
\varepsilon_{1 S}\left(M^{-1} \mathrm{~cm}^{-1}\right)=155507+6.67054 \times 10^{13} \exp \left(-\frac{E_{1 S}}{0.10551}\right)
$$

where $E_{I S}$ represent the energy corresponding to the first excitonic peak center.

The above-cited empirical formulas were obtained from batches of CdSe nanoparticles of about $11 \%$ of size dispersity, which gave rise to the first excitonic peak of a FWHM of about $120 \mathrm{meV}$. In our experiments, the FWHM of the first excitonic peaks range from 90 to $200 \mathrm{meV}$ (28 to 66 $\mathrm{nm})$ as the reaction condition changes, which could lead to some uncertainty in calculating the average particle size. The main source of error for size calculation for polydisperse particles might come from two aspects. First, the smaller particles are overshadowed by the larger ones, as the larger ones exhibit higher extinction coefficient, which leads to an overestimation of average particle size for polydisperse particles. Second, the broad $1^{\text {st }}$ and $2^{\text {nd }}$ excitonic peak partially overlap, which blueshift the $1^{\text {st }}$ excitonic peak center and leads to an underestimation of average 
particle size. Luckily, these two effects partially compensate each other, which, to our surprise, allows relatively accurate size estimation even for the most polydisperse sample we encountered, as revealed by the TEM study discussed later. To a first approximation, we assume the uncertainty in size estimation is linked to the FWHM of the $1^{\text {st }}$ excitonic peak as follows: the true average particle size is bound in the range between the sizes calculated with peak center \pm $\mathrm{FWHM} / 6$. The uncertainty calculated is in the range of 0.2 to $0.5 \mathrm{~nm}$ as the FWHM changes in the range of 28 to $66 \mathrm{~nm}$, and these numbers are used to generate the error bars in Figure 2. To verify the size estimated optically, we have performed careful TEM study on selected samples, as shown in Figure S5-S8. We found a good agreement between the size estimated by absorption peak generally and that estimated by TEM imaging: the difference is generally less than $0.3 \mathrm{~nm}$ ( $6 \%$ relative to the average size) and smaller than the uncertainty estimated from the FWHM (Table S1). We did notice an overestimation of size by the optical method for the largest particles, which are usually linked with the ripening-during-growth reaction regime (e.g. reaction $1 \mathrm{~d}, 2 \mathrm{~h}$, $3 \mathrm{c})$. This imposes some doubt on whether ripening does happen in these scenarios. Nevertheless, the size measured by TEM study of these samples are still significantly larger than the calculated "ideal" size. Therefore, our conclusion that ripening happens under these conditions still holds. Due to the existence of the uncertainty in size and particle number estimation, we would like to acknowledge that it is difficult to determine the boundaries between the three growth regimes accurately.

\section{Reaction simulations}

Model equations. The modeling approach used here was adopted from Hens et al with some modifications (see Refs. 14 and 28 in the main text). Two essential quantities including the concentration distribution of QDs $c(r, t)$ and the supersaturation $S(t)$ are calculated. The product $c(r, t) d r$ gives the concentration of QDs with a radius between $r$ and $r+d r$ at time $t$. Supersaturation is defined as $S=[M] /[M]_{0}$, where $[M]$ and $[M]_{0}$ stand for monomer concentration and bulk monomer solubility, respectively. The governing coupled differential equations are written as:

$$
\begin{aligned}
& \frac{\partial c}{\partial t}=\left.\frac{\partial c}{\partial t}\right|_{G}+\left.\frac{\partial c}{\partial t}\right|_{N}=-\frac{\partial}{\partial r}\left(j_{G} c\right)+J_{N} \delta\left(r-r_{c}\right) \\
& \frac{d S}{d t}=\frac{1}{[M]_{0}}\left\{G_{M}-\frac{4 \pi r_{c}^{3}}{3 v_{0}} J_{N}+\int_{0}^{\infty} \frac{4 \pi r^{3}}{3 v_{0}} \frac{\partial}{\partial r}\left(j_{G} c\right) d r\right\}
\end{aligned}
$$

where $j_{G}, J_{N}, r_{c}, G_{M}$, and $v_{0}$ stand for particle growth rate, nucleation rate, critical radius, monomer generation rate, and molar volume of CdSe. Equation (1) for $\partial c / \partial t$ contains the contribution from the growth of the existing particles and the nucleation of new particles. Equation (S2) for $d S / d t$ is based on the mass balance of the monomers considering monomer generation from precursor reaction and monomer consumption by particle growth and nucleation.

The expressions for the growth rate, nucleation rate, and critical radius are written as: 


$$
\begin{gathered}
j_{G}=\frac{d r}{d t}=D v_{0} N_{A}[M]_{0}\left\{\frac{S-\exp \left(\frac{2 \gamma v_{0}}{r k_{B} T}\right)}{r+\frac{D}{k_{g}^{\infty}} \exp \left(\alpha \frac{2 \gamma v_{0}}{r k_{B} T}\right)}\right\} \\
J_{N}=\frac{2 D}{v_{0}^{5 / 3}} \exp \left(-\frac{16 \pi \gamma^{3} v_{0}^{2}}{3\left(k_{B} T\right)^{3}(\ln S)^{2}}\right) \\
r_{c}=\frac{2 \gamma v_{0}}{k_{B} T \ln S}
\end{gathered}
$$

where $\mathrm{D}, \mathrm{N}_{\mathrm{A}}, \gamma, \mathrm{k}_{\mathrm{B}}, \mathrm{T}, k_{g}^{\infty}$ and $\alpha$ stand for monomer diffusion coefficient, Avogadro constant, QD surface energy per unit area, Boltzmann constant, growth temperature, and surface growth rate constant, respectively.

For $1^{\text {st }}$ order precursor conversion reactions, which were observed in this study, the monomer generation rate is expressed as:

$$
G_{M}=-\frac{d[P]}{d t}=k[P]=k[P]_{0} e^{-k t}
$$

Numerical simulation. To solve the coupled partial differential equations, the concentration distribution is discretized in a range from 0.2 to $6 \mathrm{~nm}$ into 581 elements. An absorbing boundary condition below $0.2 \mathrm{~nm}$ was used to account for the dissolution of nanocrystals into monomers. To incorporate the nucleation term, we assume the nuclei follow a Gaussian distribution with a standard deviation of $0.01 \mathrm{~nm}$, centered $0.1 \mathrm{~nm}$ above the critical radius. For seeded growth reactions, an initial concentration distribution $c(r, t=0)$ of a Gaussian function is imposed, and a 10\% relative size distribution is assumed. The parameter values used in the high-throughput simulations to generate Figure 1b and Figure 2, except for those chosen to be varied, are adopted from Hens et al., which are shown in Table S2. The discretized concentration distribution equations and the supersaturation equation are numerically solved in MATLAB in a time span from 0 to the time needed for the precursor conversion reaches $99 \%$, which is defined as the termination of the reaction.

Table S2. Parameter values used in simulations.

\begin{tabular}{|llll|}
\hline Symbol & Parameter Description & Value & Unit \\
\hline $\boldsymbol{D}$ & Monomer diffusion coefficient & $0.5 \times 10^{-9}$ & $\mathrm{~m}^{2} / \mathrm{s}$ \\
\hline $\boldsymbol{v}_{\boldsymbol{0}}$ & Monomer volume & $5.6 \times 10^{-29}$ & $\mathrm{~m}^{3}$ \\
\hline $\boldsymbol{\gamma}$ & QD surface energy & 0.2 & $\mathrm{~J} / \mathrm{m}^{2}$ \\
\hline $\boldsymbol{\alpha}$ & Transfer coefficient & 0.5 & - \\
\hline $\boldsymbol{k}_{g}^{\infty}$ & Surface growth rate constant & 50 & $\mathrm{~m} / \mathrm{s}$ \\
\hline $\boldsymbol{n}_{\boldsymbol{0}}$ & Seed particle number density & $8 \times 10^{-3}$ & $\mathrm{~mol} / \mathrm{m}^{3}$ \\
\hline $\boldsymbol{r}_{\boldsymbol{0}}$ & Average seed radius & 1.4 & $\mathrm{~nm}$ \\
\hline $\boldsymbol{P}_{\boldsymbol{0}}$ & Precursor initial concentration & 0.01 & $\mathrm{~mol} / \mathrm{m}^{3}$ \\
\hline $\boldsymbol{k}$ & Precursor reaction rate constant & Varied from $3.3 \times 10^{-5}$ to $3.3 \times 10^{-1}$ & $\mathrm{~s}^{-1}$ \\
\hline $\boldsymbol{I M} \boldsymbol{J}_{\boldsymbol{0}}$ & Monomer solubility & Varied from $1.0 \times 10^{-9}$ to $2.5 \times 10^{-7}$ & $\mathrm{~mol} / \mathrm{m}^{3}$ \\
\hline $\boldsymbol{T}$ & Growth temperature & Varied from 240 to 300 & ${ }^{\circ} \mathrm{C}$ \\
\hline
\end{tabular}


Surface energy profile modifications. To elucidate the effect of surface energy profile on the growth trajectory of an assembly of particles, we performed the simulations by imposing the three types of surface energy profiles shown in Figure S8, while kept $k,[M]_{0}$ and $T$ constant at $0.1 \mathrm{~s}^{-1}$, $4.0 \times 10^{-8} \mathrm{~mol} / \mathrm{m}^{3}$, and $300{ }^{\circ} \mathrm{C}$, respectively. The rest of parameter values are kept the same as shown in Table S2. To simplify the simulation and only focus on the size evolution by growth, we assume no nucleation in all three cases.

Discussion on oscillating surface energy profile. To rationalize an oscillating surface energy size dependence of oleic acid capped CdSe nanocrystals, we consider the surface atoms exposed as the size of the particle increase. As shown in literature, oleic acid coordinates to the cation rich (001) and (111) planes of a zinc blende CdSe lattice. Therefore, we assume the particles adopt a truncated cube shape. As the size of the particle increases, the surface exposed atoms are alternating between majorly cations to majorly anions, as shown in Figure S9a and b. As the cations can be well passivated by oleic acid, the cation exposed structures will have the lower per-atom surface energy. On the contrary, the anion exposed structures will have higher per-atom surface energy. Therefore, as the ratio between surface cations to anions oscillate with increasing size (Figure S9b), an oscillating surface energy profile can be obtained (Figure S9c). This oscillating surface energy profile is approximated with a sine function in simulation to reduce the expense of the calculation.
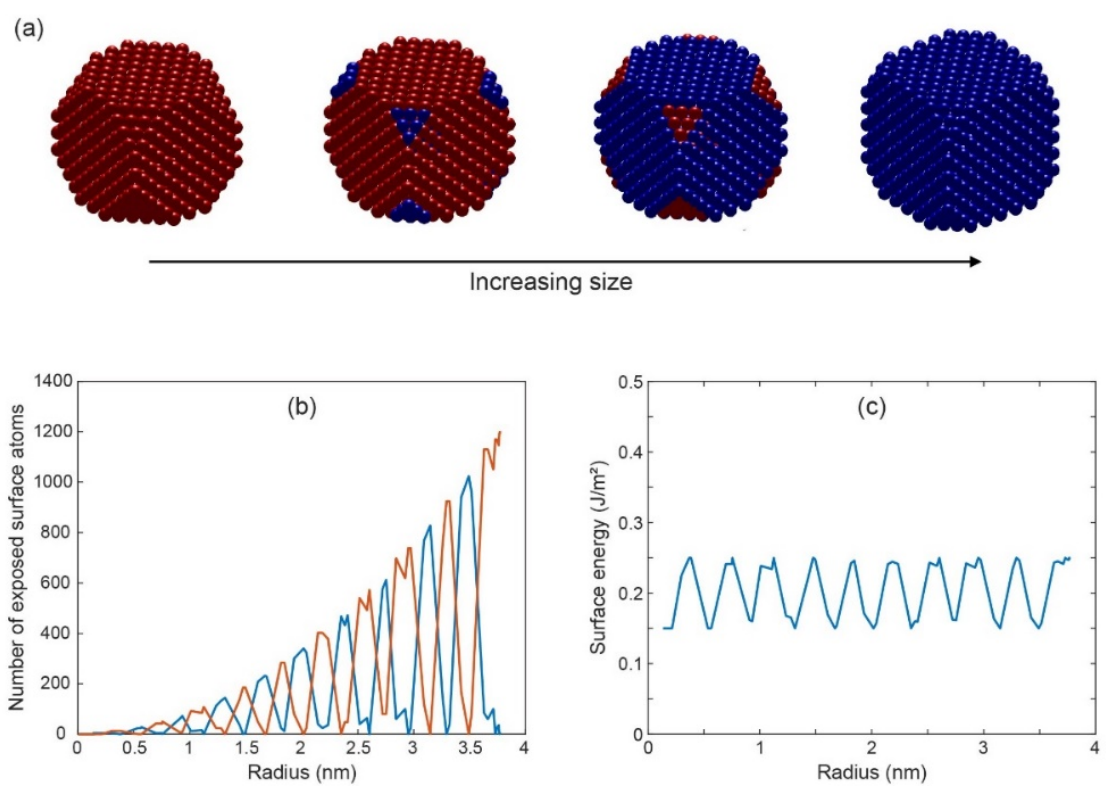

Figure S17. (a) The surface exposed atoms for a truncated cube shaped nanocrystal with a zincblende CdSe lattice as the size increases, in which red and blue colored spheres represent cation and anions, respectively. (b) The number of cations and anions exposed as a function of particle effective radius. (c) The calculated surface energy as a function of particle effective radius exhibiting oscillating characteristic. 